\section{Assessing power function relationships in magnitude estimation}

\author{
BARSHA J. COLEMAN, RICHARD G. GRAF, \\ and EDWARD F. ALF \\ San Diego State University, San Diego, California 92182
}

When magnitude estimation procedures are used in psychophysical experiments, the subject is presented several stimuli of varying magnitudes and is requested to supply a number that matches the magnitude of each stimulus. Since Stevens (1957) first suggested these procedures, it has been widely believed that the relationship between the stimulus magnitude values and the response magnitude values is properly described by a power function. Sometimes the adequacy of the power function model is assessed by examining the correlation between the logarithm of the stimulus and the logarithm of the response.

The work of several investigators, however, should lead to questioning the advisability of testing the goodness of fit of the power function model by examining the magnitude and significance of this correlation. The criticisms that have been made apply not only to the power function model, but to logarithmic and exponential models as well. In fact, the criticisms apply to the use of the correlation coefficient to measure the goodness of the fit of any model to magnitude estimation data or to any cross-modal matching data in general.

Yntema and Torgerson (1961), for example, make the general point that the linear model often provides a good approximation, even when there are significant curvilinear or configural relationships in the data. Anderson and Shanteau (1977) and Birnbaum (1973) have pointed out that any monotone function will have a large linear component. Good (1972) has shown that, even for very nonlinear monotone functions, the linear correlation coefficient can be quite close to 1.00 .

It is an elementary algebraic demonstration that for $\mathrm{n}$ equally spaced values of $\mathrm{x}$, if $\mathrm{y}$ is any monotone increasing function of $x$, then the lower bound for the linear correlation, $r$, between $x$ and $y$ is $r=$ $[3 /(n+1)]^{1 / 2}$. Thus, if the number of data points is small, the correlation between $\mathrm{x}$ and $\mathrm{y}$ is bound to be fairly high, even for an inappropriate model.

The first author is now at the University of California, San Diego. A version of this paper was presented at the Convention of the Western Psychological Association, San Diego, April 1979, and was supported in part by Grant RO3MH31598 from the National Institute of Mental Health. The authors are indebted to Dr. Norman H. Anderson, University of California, San Diego, for outlining this problem and the general method of solution.
Thus, the size and significance of the correlation coefficient may be largely tangential to the question of whether a given model is appropriate. A significant correlation simply allows us to reject the null hypothesis that the population correlation is zero. Since the lower bound for this correlation will generally be greater than zero, the null hypothesis that the population correlation is zero will be inappropriate. And since all monotonic increasing models will tend to have high correlations, a high and significant correlation does not tell us whether our model, or some other model, is appropriate for the data.

A proper goodness-of-fit test is one that tests the significance of the deviations from the model being tested. In order to have a proper error term for the goodness-of-fit test, it is desirable to have data sets with replications at every value of stimulus magnitude being tested. The appendix presents guidelines for such an analysis of variance for a power model and illustrates that the model may not fit the data adequately, even when the correlation equals .98 .

The model assumed in this analysis is as follows. The response of any one individual for a particular stimulus can be written as

$$
Y_{i j}=a+b\left(x_{i j}\right)^{n}+A_{i}+E_{i j},
$$

where $y_{i j}$ is the response magnitude for the $j^{\text {th }}$ replication of the stimulus at the $i^{\text {th }}$ intensity, the first two terms on the right of the equation are the power law being tested, $A_{i}$ is the deviation from the power law at the $i^{\text {th }}$ stimulus intensity, and $E_{i j}$ is random error. The power law is completely correct only if all $\mathbf{A}_{\mathrm{i}}=\mathbf{0}$, signifying no departures from the model. Thus, the null hypothesis of interest is that the variance of the $A_{i}$ values is zero. The $E_{i j}$ are assumed to be normally distributed, homoscedastic, and mutually independent. The independence of the errors must be assured by the experimental design that is used in gathering the data. However, the failure to meet the first two assumptions will not substantially affect the results of the analysis of variance (Scheffé, 1959).

Least-squares estimates of the parameters $a, b$, and $\mathrm{n}$ of the power model can be found using the method given by Snedecor and Cochran (1967, pp. 465-471) for estimating the constants in equations that are nonlinear in some of the parameters. These estimates and the stimulus values are then used to generate the response values at each stimulus intensity that would be predicted by the model.

The total response variability may be divided into three additive parts: (1) the variability accounted for by Stevens' power law, (2) the residual variability among the treatment means, and (3) the withintreatments variability. 
The significance of the power function component of the between-treatments variability is tested by the $F$ ratio obtained by dividing the power function mean square by the within-treatments mean square. Departures from the model may be tested for significance by the $F$ ratio obtained by dividing the mean square for the residual variability among treatments by the within-treatments mean square.

In the example cited in the appendix, the power function component in the data is highly significant. Also, it accounts for about $98 \%$ of the variance in the subject's responses.

The departures from the power model account for a little less than $.5 \%$ of the variance in the subject's responses, but these departures are highly significant. Thus, even though a power function may account for a large portion of the total subject response variance, it is still possible that small, though significant, departures from the power model may prove it inadequate to explain the variability in the data.

A distinction should be made between the theoretical and the practical uses of a model. From a practical point of view, a model accounting for about $\mathbf{9 8 \%}$ of the variability in the data may be highly use- ful for descriptive or predictive purposes. From a theoretical point of view, however, even if the significant departures from the model are very small, it signifies there are small, but significant, differences between the judgments of the subject and those that would be predicted by the model. It is by exploring the nature of these differences that more appropriate models may eventually be found.

\section{REFERENCES}

Anderson, N. H., \& Shanteau, J. Weak inference with linear models. Psychological Bulletin, 1977, 84, 1155-1170.

Birnbaum, M. H. The devil rides again: Correlation as an index of fit. Psychological Bulletin, 1973, 81, 854-859.

Good, I. J. Correlation for power functions, Biometrics, 1972, 28, $1127-1129$.

SCHEFFÉ, H. The analysis of variance. New York: Wiley, 1959.

Snedecon, G. W., \& Cochran, W. G. Statistical methods. Ames: Iowa State University Press, 1967.

Stevens, S. S. On the psychophysical law. Psychological Review, $1957,64,377-411$.

YNTema, D. B., \& Tongerson, W. S. Man-computer cooperation in decisions requiring common sense. IRE Transactions of the Professional Group on Human Factors in Electronics, 1961, HFE 2(1), 20-26.

\section{APPENDIX}

\section{Goodness-of-Fit Tests for Stevens' Power Law}

Stevens' power function is of the form:

$$
\tilde{y}=a+b x^{n} .
$$

The constants $\mathrm{a}, \mathrm{b}$, and $\mathrm{n}$ can be determined by the method of least squares. Data for a single subject are presented in Table A. For the data in Table A, the best fitting Stevens power function is:

$$
\tilde{y}=(-54.86378842)+(52.68977154) x^{(.0128)} \text {. }
$$

By substituting the five stimulus values of the experiment into this equation, we obtain the predicted mean $y$ values

Table A

Observations on a Dependent Variable, $y$, for a Single Subject at Five Levels of an Independent Variable, $x$, With Five Replications at Each Level

\begin{tabular}{lccccc}
\hline $\begin{array}{c}\text { Replica- } \\
\text { tion }\end{array}$ & 5.5 & 13.7 & 27.2 & 46.8 & 73.4 \\
\hline 1 & -1.0764 & -.4361 & .2907 & .5412 & .6806 \\
2 & -.9585 & -.4493 & .1154 & .6045 & .6879 \\
3 & -1.0261 & -.5209 & .2081 & .5674 & .7716 \\
4 & -.9737 & -.4960 & .1387 & .5755 & .7555 \\
5 & -.8368 & -.5764 & .0739 & .5340 & .8053 \\
$\Sigma y_{\mathbf{i}}$ & -4.8715 & -2.4787 & .8268 & 2.8226 & 3.7009 \\
$\Sigma y_{\mathbf{i}}^{2}$ & 4.7786 & 1.2416 & .1658 & 1.5966 & 2.7511 \\
\hline
\end{tabular}

Note-Across all groups, $\Sigma y=.0001$ and $\Sigma y^{2}=10.5337$. for each treatment. These predicted mean values follow the actual mean $y$ values very well, although there are deviations.

The total response variability may be divided into three additive parts: (1) the variability accounted for by Stevens' power law, (2) the residual variability associated with the treatment means, and (3) the within-treatments variability.

Using the values predicted from Stevens' power equation, $\tilde{y}$, and the treatment means, $\overline{\mathbf{y}}$, we find:

$$
\mathrm{r}_{\tilde{\mathrm{y}} \overline{\mathrm{y}}}^{2}=.985818668 \text {. }
$$

From this, we find:

$$
\begin{aligned}
\text { Stevens' SS } & =(\text { treatments } S S)\left(r_{\tilde{y} \tilde{y}}^{2}\right) \\
& =(10.4446)(.985818668) \cong 10.2765 ;
\end{aligned}
$$

Residual SS $=$ treatments SS - Stevens' SS $\cong .1481$.

We may then write Table B, which is the summary table for this model.

The significance of Stevens' power function component of the between-treatments variability is tested with the F ratio:

$$
\mathrm{F}(\text { Stevens })=\frac{\text { Stevens' MS }}{\text { Within MS }}=1144.07
$$


Table B

Summary Table for Stevens' Power Analysis

\begin{tabular}{lrrrr}
\hline Source & \multicolumn{1}{c}{ SS } & df & \multicolumn{1}{c}{ MS } & \multicolumn{1}{c}{ F } \\
\hline Stevens & 10.2965 & 2 & 5.1483 & 1144.07 \\
Residual & .1481 & 2 & .0741 & 16.47 \\
Within & .0891 & 20 & .0045 & \\
Total & 10.5337 & 24 & & \\
\hline
\end{tabular}

With 2 and 20 degrees of freedom, this $F$ value is highly significant.

The magnitude of association for Stevens' power function is tested by the eta squared value:

$$
\eta_{\mathrm{yx}}^{2}(\text { Stevens })=\frac{\text { Stevens' SS }}{\text { Total SS }}=.9775 .
$$

Departures from Stevens' model are tested for significance by:

$$
F(\text { Residual })=\frac{\text { Residual MS }}{\text { Within MS }}=16.47 .
$$

With 2 and 20 degrees of freedom, this $F$ value exceeds the critical value at the $1 \%$ level.

The magnitude of departures from Stevens' power function is assessed by means of eta squared for the residual sum of squares, computed by:

$$
\eta_{\mathrm{yx}}^{2}(\text { Residual })=\frac{\text { Residual SS }}{\text { Total SS }}=.0141
$$

